\title{
A finite difference scheme for a macroscopic traffic flow model based on a nonlin- ear density-velocity relationship
}

\author{
M. H. Kabir ${ }^{\mathrm{a}}$, A. Afroz ${ }^{\mathrm{b}}$ and L. S. Andallah ${ }^{\mathrm{a}}$

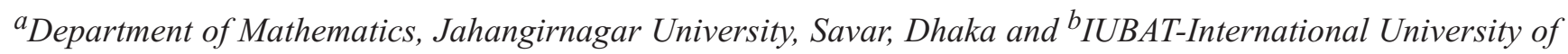 \\ Business Agriculture and Technology, Uttara, Dhaka
}

\begin{abstract}
We consider a macroscopic traffic flow model tagged on a closure nonlinear density-velocity relationship yielding a quasi-linear first order (hyperbolic) partial differential equation (PDE) as an initial boundary value problem (IBVP). We present the analytic solution of the PDE which is in implicit form. We describe the derivation of a finite difference scheme of the IBVP which is a first order explicit upwind difference scheme. We establish the well-posed-ness and stability condition of the finite difference scheme. To implement the numerical scheme we develop computer program using MATLAB programming language in order to verify some qualitative behaviors for various traffic parameters.
\end{abstract}

Key words: Finite difference scheme, Macroscopic, Well-posed-ness, Stability, etc

\section{Introduction}

Traffic flow has been studied since the first half of the last century. It is growing field of study because it's many applications in transportation and urban planning, among others. The macroscopic approach is analogous to theories of fluid dynamics or continuum hypothesis. Macroscopic traffic flow models are characterized by representations of traffic flow in terms of aggregate measures such as flux, space mean speed, and density. Unlike microscopic models which represent individual vehicle movements, macroscopic models sacrifice a great deal of detail but gain by way of efficiency an ability to deal with problems of much larger scope.

The aims of this analysis are principally represented by the maximization of vehicles flow, and the minimization of traffic congestion, accidents and pollutions etc.

In this paper (section 2), we consider a macroscopic fluiddynamic model based on Habermann, Klar (2004). As presented in Kabir (2004), we study numerical method for first order nonlinear PDE from Leveque (1992), Larsson amd Thomee (1997), Morton and Mayers (1996) and based on these, we present the derivation a finite difference scheme for our traffic flow model as an IBVP which has been presented in Section 3. In section 5, we establish the wellposed-ness and stability condition of the finite difference scheme which has been presented in Kabir (2006). We devel op computer program in MATLAB for the implementation of numerical scheme in order to verify some qualitative traffic flow behavior for various traffic flow parameters. The algorithm of this program is presented here.

\section{Macroscopic Traffic Flow Model}

The macroscopic traffic model developed first by Lighthill and Whitham and Richard shortly called LWR model was most suitable for correct description of traffic flow. In this model, vehicles in traffic flow are considered as particles in fluid: further the behavior of traffic flow is modeled by the method of fluid dynamics and formulated by hyperbolic partial differential equation (PDE).

The macroscopic traffic flow model is used to study traffic flow by collective variables such as traffic flow rate (flux) $q(x, t)$, traffic speed $V(x, t)$ and traffic density $\rho(x, t)$, all of which are functions of space, $\mathrm{x} \varepsilon R$ and time, $\mathrm{x} \varepsilon R^{+}$. The most well-known LWR model is formulated by employing the conservation equation

$$
\frac{\partial \rho}{\partial t}+\frac{\partial q(\rho)}{\partial x}=0
$$

The LWR model comes from two facts and one assumption. 
The two facts are

a) On a homogeneous road without sources and sinks, the number of vehicles on the road is conserved and $V$

b) The flux, $q$ is a product of density, $\rho$ and speed .

The assumption is about the existence of a unique relation between speed and density. In this thesis, we use a non-linear velocity-density relationship of the form

$V(\rho)=V_{\max }\left(1-\left(\frac{\rho}{\rho_{\max }}\right)^{2}\right)$

then the flux is of the form

$q(\rho)=\rho V(\rho)=\rho \cdot V_{\max }\left(1-\left(\frac{\rho}{\rho_{\max }}\right)^{2}\right)$

Finally (2.1) gives a quasi-linear hyperbolic partial differential equation of the form

$\frac{\partial \rho}{\partial t}+\frac{\partial}{\partial x}\left(V_{\max }\left(\rho-\frac{\rho^{3}}{\rho_{\max }^{2}}\right)\right)=0$

The nonlinear density-velocity relationship (2.2) satisfies the following qualitative physical properties

$V(\rho=0)=V_{\max }$

$\frac{d V}{d \rho} \leq 0$

$V\left(\rho=\rho_{\max }\right)=0$

The corresponding flux-density relationship is parabolic and concave and satisfies the qualitative properties as shown as

$q(0)=0$

$V\left(\rho_{\max }\right)=0$

$q\left(\rho_{\max }\right)=\rho_{\max } V\left(\rho_{\max }\right)=0$

It follows that the maximum traffic flow is achieved at a point of the fundamental diagram for $\rho=\frac{\rho_{\max }}{\sqrt{3}}$ and in this
case, the maximum flow is

$q_{\max }=\frac{2}{3 \sqrt{3}} V_{\max } \rho_{\max }$
Analytic Solution of the Traffic Flow Model

The non-linear PDE (2.4) mentioned at section 2 can be solved if we know the traffic density at a given initial time, i.e., if we have the traffic density at a given initial time $t_{0}$, we can predict the traffic density for all future time $t \geq t_{0}$, in principle.

Then we are required to solve an initial value problem (IVP) of the form

$\frac{\partial \rho}{\partial t}+\frac{\partial}{\partial x}\left(\rho \cdot V_{\max }\left(1-\frac{\rho^{2}}{\rho_{\max }^{2}}\right)\right)=0$

$\rho\left(t_{0}, x\right)=\rho_{0}(x)$

The IVP (3.1) can be solved by the method of characteristics as follows:

The PDE in (3.1) may be written as

$\frac{\partial \rho}{\partial t}+\frac{\partial q(\rho)}{\partial x}=0$

where $q(\rho)=\rho \cdot V_{\max }\left(1-\frac{\rho^{2}}{\rho_{\max }^{2}}\right)$

$\Rightarrow \frac{\partial \rho}{\partial t}+\frac{d q}{d \rho} \frac{\partial \rho}{\partial x}=0$

$\Rightarrow \frac{\partial \rho}{\partial t}+V_{\max }\left(1-\frac{3 \rho^{2}}{\rho_{\max }^{2}}\right) \frac{\partial \rho}{\partial x}=0$

Now

$\frac{d \rho}{d t}=\frac{\partial \rho}{\partial t}+\frac{\partial \rho}{\partial x} \frac{d x}{d t}=0$

Where

$\frac{d x}{d t}=V_{\max }\left(1-\frac{3 \rho^{2}}{\rho_{\max }^{2}}\right)$

Equations (3.2) and (3.3) give

$x(t)=V_{\max }\left(1-\frac{3 \rho^{2}}{\rho_{\max }^{2}}\right) t+x^{0}$

, which is the characteristics of the IVP (3.1).

Now from equation (3.2) we have

$\frac{d \rho}{d t}=0$

$\therefore \rho(x, t)=c$ 
Since the characteristics through $(x, t)$ also passes through $\left(x^{0}, 0\right)$ and $\rho(x, t)=c$ is constant on this curve, so we use the initial condition to write

$c=\rho(x, t)=\rho\left(x^{0}, 0\right)=\rho_{0}\left(x^{0}\right)$

Equation (3.5) and (3.6) yield

$\rho(x, t)=\rho_{0}\left(x^{0}\right)$

Using equation (3.4), (3.7) takes the form

$\rho(x, t)=\rho_{0}\left(x-V_{\max }\left(1-\frac{3 \rho^{2}}{\rho_{\max }^{2}}\right) t\right)$

This is the analytic solution of the IVP (3.1).

This solution is in implicit form because $\rho$ also appears in right side. It is much more difficult to transform into explicit form. Therefore, there is a demand of some efficient numerical techniques for solving the IVP (3.1).

\section{Numerical Schemes for Traffic Flow Model}

As mentioned at section 2, traffic model yields

$$
\begin{aligned}
& \frac{\partial \rho}{\partial t}+\frac{\partial}{\partial x}\left(\rho \cdot V_{\max }\left(1-\left(\frac{\rho}{\rho_{\max }}\right)^{2}\right)\right)=0 \\
& \text { where } x \in(a, b) ; t \in(0, T)
\end{aligned}
$$

The initial and boundary conditions are given by

$\rho(x, 0)=\rho_{0}(x)$ and $\rho(a, t)=\rho_{\mathrm{a}}(t)$

The equations (4.1) and (4.2) produce an initial boundary value problem (IBVP) which is well-posed for left hand boundary if velocity, $V_{\max }>0$ and for the right hand boundary if velocity, $V_{\max }<0$

Equation (4.1) may be transformed as

$$
\frac{\partial \rho}{\partial t}+\frac{\partial q(\rho)}{\partial x}=0
$$

where $q(\rho)=\left(\rho \cdot V_{\max }\left(1-\left(\frac{\rho}{\rho_{\max }}\right)^{2}\right)\right)$

Firstly, we would like to derive an appropriate numerical scheme for the equation (4.3).
In the case of linear equation (4.1), $f_{i+\frac{1}{2}}^{n+\frac{1}{2}}=V_{\max } \rho$

In order to develop the scheme, we discretize the space and time. The discretization of $\frac{\partial \rho(x, t)}{\partial t}$ is obtained by first order forward difference in time and the discretization of $\frac{\partial \rho(x, t)}{\partial t}$ is obtained by first order backward difference in space.

The possible finite difference approximations for $\frac{\partial \rho}{\partial t}$ and $\frac{\partial \rho}{\partial x}$ :

Forward difference in time:

From Taylor's series we write

$\rho(x, t+h)=\rho(x, t)+h \frac{\partial \rho(x, t)}{\partial t}+\frac{h^{2}}{2 !} \frac{\partial^{2} \rho(x, t)}{\partial t^{2}}+\ldots$

$\Rightarrow \frac{\partial \rho(x, t)}{\partial t}=\frac{\rho(x, t+h)-\rho(x, t)}{h}-o(h)$

$\therefore \frac{\partial \rho(x, t)}{\partial t} \approx \frac{\rho(x, t+h)-\rho(x, t)}{h}$

Similarly, backward difference in space

$\frac{\partial \rho(x, t)}{\partial x} \approx \frac{\rho(x, t)-\rho(x-k, t)}{k}$

We assume the uniform grid spacing with step size $h$ and $k$ for time and space respectively $t^{\mathrm{n}+1}=t^{\mathrm{n}}+h$ and $x_{i+1}=x_{i}+k$.

We also write $\rho^{n}{ }_{i}$ for $\rho(x, t)$ in equation (4.4) and (4.5).

Now equation (4.3) takes the form

$\frac{\rho_{i}^{n+1}-\rho_{i}^{n}}{\Delta t^{n+\frac{1}{2}}}+\frac{q_{i}^{n}-q_{i-1}^{n}}{\Delta x_{i}}=0$

$\Rightarrow \rho_{i}^{n+1}=\rho_{i}^{n}-\frac{\Delta t^{n+\frac{1}{2}}}{\Delta x_{i}}\left[q_{i}^{n}-q_{i-1}^{n}\right]$

where $q_{i}^{n}=\rho_{i}^{n} V_{\max }\left(1-\left(\frac{\rho_{i}^{n}}{\rho_{\max }}\right)^{2}\right)$

This is the explicit upwind difference scheme for the equation (4.1).

Therefore, equation (4.6) leads the desired scheme for the traffic model. 
The stencil for the explicit upwind difference scheme (4.6) is presented below

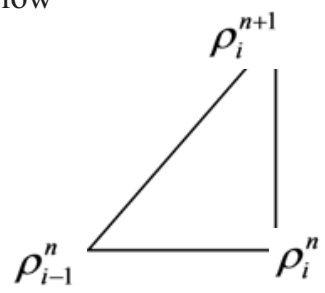

Well-posed-ness and Stability Condition

It is not straight forward to implement the explicit upwind difference scheme. One needs to ensure the well-posed-ness condition

$$
\begin{array}{r}
\frac{\partial \rho}{\partial t}+\frac{\partial q(\rho)}{\partial x}=( \\
\text { where } q(\rho)=\left(\rho \cdot V_{\max }(1)\right.
\end{array}
$$

\section{Proposition}

The well-posed-ness and stability condition of the explicit upwind difference scheme (4.6) is given by the simultaneous conditions respectively

$\rho_{\max }=c \max _{k} \rho_{0}\left(x_{k}\right) ; c \geq \sqrt{3}$ and $\gamma=\frac{V_{\max } \Delta t}{\Delta x} \leq 1$

\section{Proof}

We recall the traffic flow model as

$\frac{\partial \rho}{\partial t}+\frac{\partial q(\rho)}{\partial x}=\mathbf{0}$

where $q(\rho)=\rho V(\rho)=\rho V_{\max }\left(1-\frac{\rho^{2}}{\rho_{\max }^{2}}\right)$

PDE (5.4) can be written as

$\frac{\partial \rho}{\partial t}+q^{\prime}(\rho) \frac{\partial \rho}{\partial x}=0$

where $q^{\prime}(\rho)=V_{\max }\left(1-\frac{3 \rho^{2}}{\rho_{\max }^{2}}\right)$

The explicit upwind difference scheme (4.6) takes the form

$$
\begin{aligned}
& \rho_{i}^{n+1}=\rho_{i}^{n}-q^{\prime}\left(\rho_{i}^{n}\right) \frac{\Delta t^{n+\frac{1}{2}}}{\Delta x_{i}}\left[\rho_{i}^{n}-\rho_{i-1}^{n}\right] \\
\Rightarrow & \rho_{i}^{n+1}=(1-\lambda) \rho_{i}^{n}+\lambda \rho_{i-1}^{n}
\end{aligned}
$$

where $\lambda:=q^{\prime}(\rho) \frac{\Delta t^{n+\frac{1}{2}}}{\Delta x_{i}}$

The equation (5.6) implies that if $\lambda \leq 1$, the new solution is a convex combination of the two previous solutions. That is the solution at new time step $(n+1)$ at a spatial-node $i$, is an average of the solutions at the previous time-step at the spatial-nodes $i$ and $i-1$. This means that the extreme value of the new solution is the average of the extreme values of the previous two solutions at the two consecutive nodes. Therefore, the new solution continuously dependent on the initial value $\rho_{i}^{0} i=1, \ldots, M$ and the explicit upwind difference scheme is stable for

$\lambda:=q^{\prime}\left(\rho_{i}^{n}\right) \frac{\Delta t^{n+\frac{1}{2}}}{\Delta x_{i}} \leq 1$

Then with the aid of the condition (5.3) we write,

$\gamma_{i}^{n+\frac{1}{2}}:=\frac{V_{\max } \Delta t^{n+\frac{1}{2}}}{\Delta x_{i}} \leq 1$

which is the stability condition that can be implemented in the computer program very easily.

Hence whenever we employ the stability condition (5.8), the well-posed-ness condition (5.2) can be guaranteed instantaneously by choosing

$$
\rho_{\max }=c \max _{k} \rho_{0}\left(x_{k}\right) ; c \geq \sqrt{3}
$$

\section{Numerical experiments and results}

In order to implement the numerical scheme we develop a computer program and perform numerical experiments as presented below.

To find the numerical solution of the IBVP (2.5) we have to store some variables and values which are presented in the following algorithm.

Algorithm for the numerical solution of traffic flow model:

\section{Input}

$n x$ and $n t$ the number of spatial and temporal grid points respectively.

$t f$, the right end point of $[0, T]$.

$x d$, the right end point of $[0, b]$.

$\rho 0$, the initial density, apply as a initial condition. 
$\rho a$, the vehicles at starting point, apply as a boundary value.

$V \max$, maximum velocity.

\section{Output}

$\rho(x, t)$ the solution matrix.

Initialization

$d t=\frac{T-0}{n t}$, the temporal grid size

$d x=\frac{b-0}{n x}$, the spatial grid size.

$g m=V \max * \frac{d t}{d x}$, the courant number.

$g m a=1-g m$

$c=10$, a constant. $(c \geq \sqrt{3})$

$\rho_{\max }=\max (\rho 0) * c$, maximum density.

Step 1: Calculation for upwind scheme

For $i=1$ to $n t$

For $j=2$ to $n x+1$

$$
\begin{aligned}
& \rho(i+1, j)=g m a^{*} \rho(i, j)+g m^{*} \rho(i, j-1)+ \\
& \left((d t / d x)^{*}\left(V \max / \rho \max ^{\wedge} 2\right)\right) \\
& \left.{ }^{*}\left(\rho(i, j) \text {. }^{\wedge}-\rho(i, j-1)\right)^{\wedge} 3\right) \\
& \quad \text { End } \\
& \quad \text { End }
\end{aligned}
$$

\section{Step 2: Calculation for velocity}

For $i=1$ to $n t$

$$
v(i)=V \max ^{*}\left(1-\rho(i) .^{\wedge} 2 / \rho \max . \wedge 2\right)
$$

End

\section{Step 3: Calculation for flux}

For $i=1$ to $n t$

$$
q(i)=V \max ^{*}\left(\rho(i)-\rho(i) .^{\wedge} 3 / \rho \max .^{\wedge} 2\right)
$$

End

\section{Step 4: Output $\rho(x, t)$}

\section{Step 5: Graph Presentation}

\section{Step 6: Stop}

To estimate the error, we consider the analytic colution ( 28$)$ determined in section 3 with initial condition $\rho_{0}(x)=\frac{1}{2} x$, chosen arbitrarily.

$$
\begin{aligned}
& \Rightarrow 3 \rho^{2} t V_{\max }-2 \rho \rho_{\max }^{2}+x t \rho_{\max }^{2}-V_{\max } \rho_{\max }^{2} t=0 \\
& \therefore \rho(t, x)=\frac{2 \rho_{\max }^{2} \pm \sqrt{4 \rho_{\max }^{4}-12 t V_{\max }\left(x t \rho_{\max }^{2}-V_{\max } \rho_{\max }^{2} t\right)}}{6 t V_{\max }} \\
& \Rightarrow \rho(t, x)=\frac{\rho_{\max }^{2} \pm \rho_{\max }^{2} \sqrt{\rho_{\max }^{2}-3 t^{2} V_{\max } x-3 V_{\max }^{2} t^{2}}}{3 t V_{\max }}
\end{aligned}
$$

that is

$$
\begin{aligned}
& \rho(t, x)=\frac{\rho_{\max }^{2}+\rho_{\max }^{2} \sqrt{\rho_{\max }^{2}-3 t^{2} V_{\max } x-3 V_{\max }^{2} t^{2}}}{3 t V_{\max }}, \\
& \frac{\rho_{\max }^{2}-\rho_{\max }^{2} \sqrt{\rho_{\max }^{2}-3 t^{2} V_{\max } x-3 V_{\max }^{2} t^{2}}}{3 t V_{\max }}
\end{aligned}
$$

Since $\rho_{\max }$ is maximum density so,

$$
\rho_{\max }^{2}+\rho_{\max }^{2} \sqrt{\rho_{\max }^{2}-3 t^{2} V_{\max } x-3 V_{\max }^{2} t^{2}} \text { gives a value }
$$

which exceeds the maximum density. That's why

we cannot accept this.

Finally, the analytic solution is

$$
\rho(t, x)=\frac{\rho_{\max }^{2}-\rho_{\max }^{2} \sqrt{\rho_{\max }^{2}-3 t^{2} V_{\max } x-3 V_{\max }^{2} t^{2}}}{3 t V_{\max }}
$$

In that case, we recommend the respective

boundary condition

$$
\rho_{a}(t)=\rho\left(t, x_{a}\right)=\frac{\rho_{\max }^{2}-\rho_{\max }^{2} \sqrt{\rho_{\max }^{2}-3 t^{2} V_{\max } x-3 V_{\max }^{2} t^{2}}}{3 t V_{\max }}
$$

For the above initial and boundary condition with maximum speed $\quad V_{\max }=0.167=60 \mathrm{~km} / \mathrm{hour}$ satisfying the well-posed-ness condition $\rho_{\max }=5 \max _{k} \rho_{0}\left(x_{k}\right)=250 / \mathrm{km}$ in the spatial domain $[0 \mathrm{~km}, 10 \mathrm{~km}]$, we perform the numerical experiment for 9 minutes in $\Delta t=0.1$ time steps for a highway of $10 \mathrm{~km}$ in 101 grid points with step size $\Delta x=100$ meters $=0.25$ which guarantees the stability condition $\gamma=0.0668<1$. We compute the relative error in $L_{1}$ norm defined by

$\|e\|_{1}:=\frac{\left\|\rho_{e}-\rho_{n}\right\|_{1}}{\left\|\rho_{e}\right\|_{1}}$ 
for all time where $\rho_{e}$ is the analytic solution (6.1) and $\rho_{n}$ is the numerical solution computed by the finite difference scheme. In the fig 6.1, the analytic and numerical solution is presented. Figure (6.2) represents that the relative error remains below 0.0032 which is acceptable
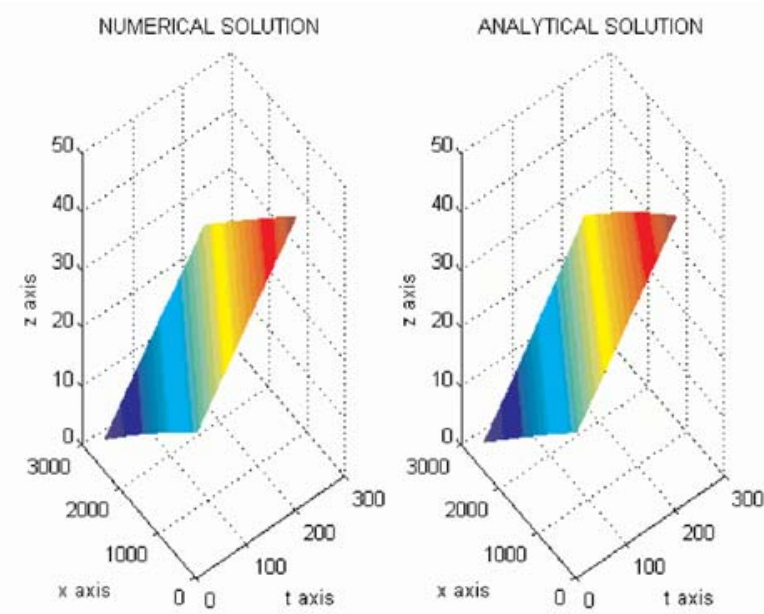

Fig 6.1: Analytic \& Numerical Solution

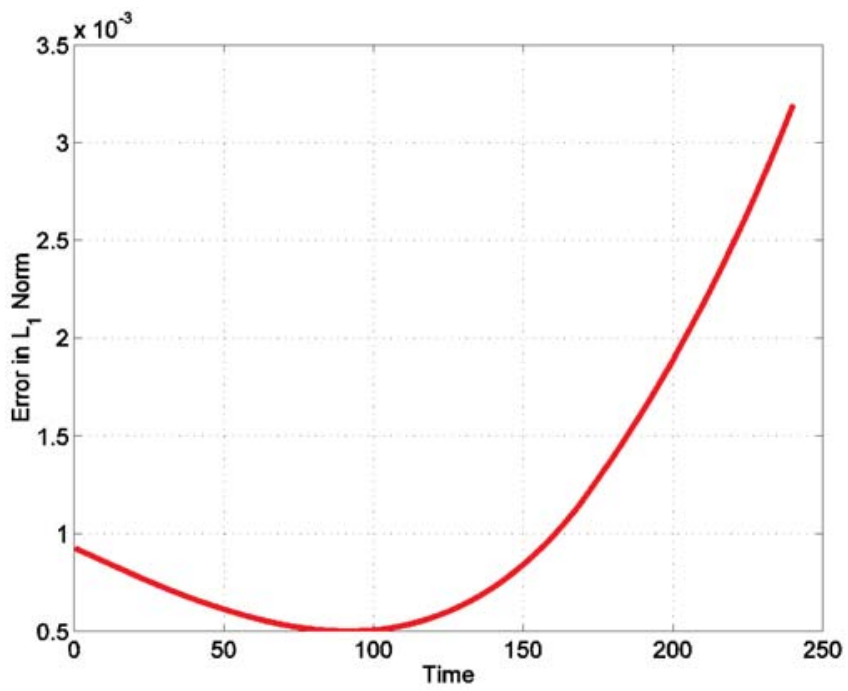

Fig. 6.2: Relative Error

Now consider the initial density as shown in fig 6.3 and carry out numerical computation in the spatial domain $[0,10]$ in $\mathrm{km}$. Fig 6.4 presents the density profile for $3 \mathrm{~min}, 6 \mathrm{~min}$ and 9 minutes respectively.

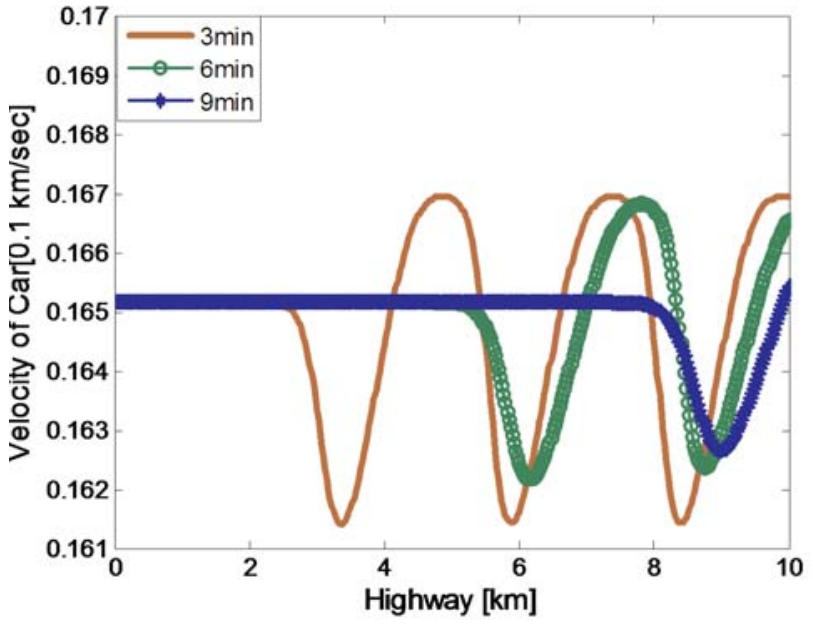

Fig. 6.3: Initial density

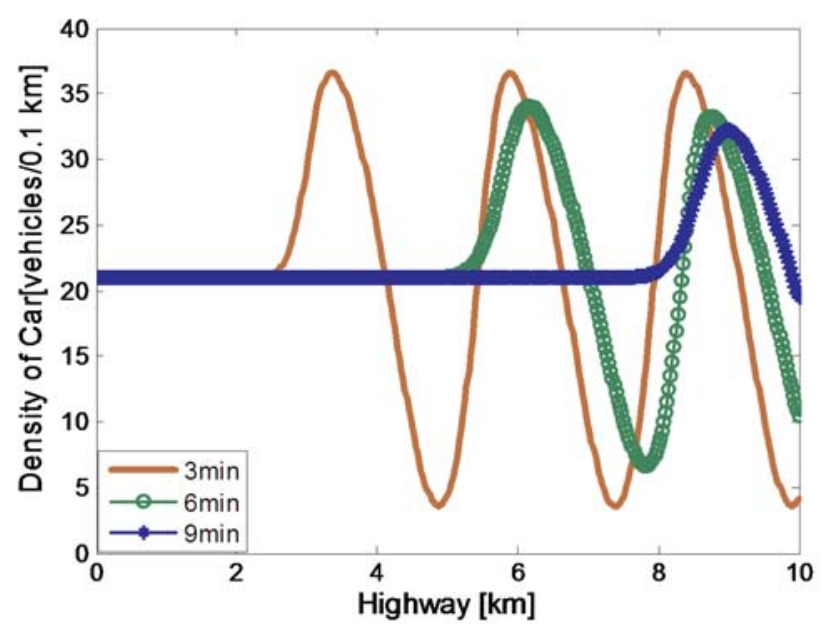

Fig 6.4: Density profile

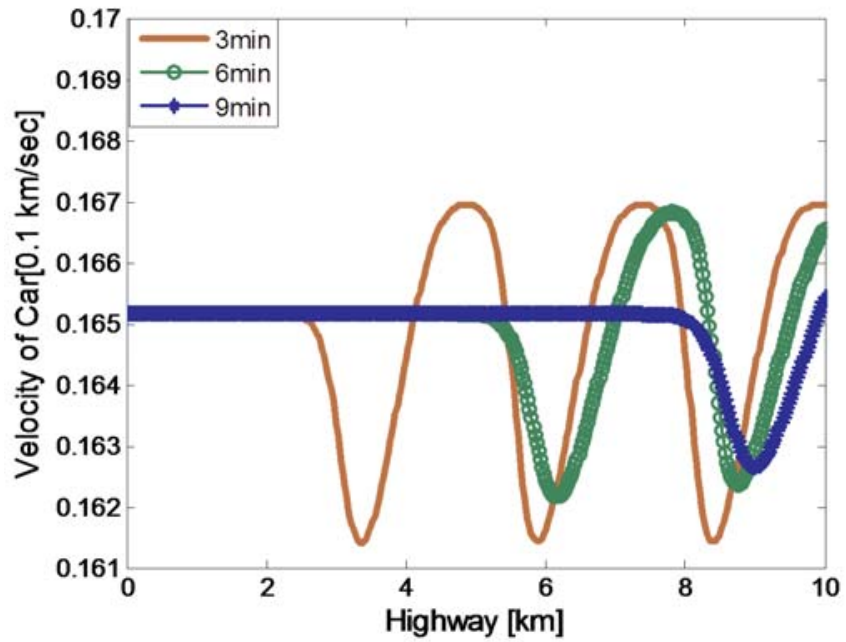

Fig 6.5: Velocity profile 
Fig 6.5 and 6.6 represent the respective computed velocity profile and flow profile according to the certain points of the highway.

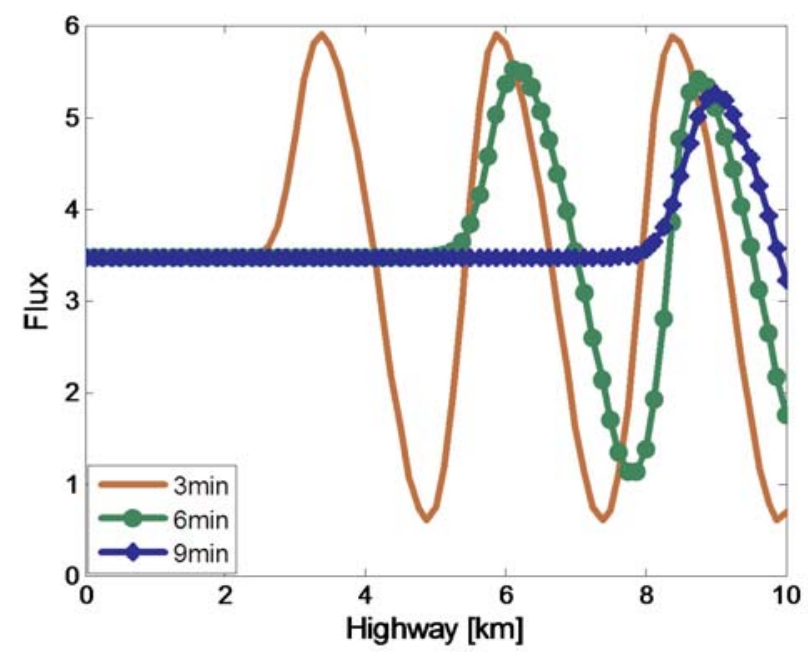

Fig 6.6: Flux profile

\section{Conclusion}

We have presented the analytic solution of a macroscopic traffic flow model by the method of characteristics which is in implicit form. We have derived the explicit upwind difference scheme for our considered traffic flow model. We have established the well-posed-ness and stability condition of the numerical scheme. The relative error between the Numerical solution and the Analytic solution has been computed using norm and the relative error is acceptable. Computer program in MATLAB has been used to predict density profile and velocity profile as well as flux profile for the implementation of the explicit upwind difference scheme. The algorithm of the computer program has been presented. This study can be expanded for multi-lane traffic flow model which will be our future interest.

\section{References}

Axel Klar, Reinhart D. Kuhne and Raimurd Wegener (2004). Mathematical models for Vehicular Traffic", Technical University of Kaiserlautern, Germany.

Kabir MH (2006). Traffic Flow Simulation by using a Mathematical Model based on a Non- linear VelocityDensity Function", M.S. Thesis 2006, Department of Mathematics, Jahangirnagar University, Dhaka

Kabir MH, Gani MO and Andallah LS (2010). Numerical Simulation of a Mathematical Traffic flow Model based on a nonlinear velocity-density function, Journal of Bangladesh Academy of Sciences, 34: Number 1, June, ISSN.

Morton KW and Mayers DF (1996). Numerical Solution of Partial Differential Equations, Cambridge University Press.

Randall J and Le Veque (1992). Numerical Methods for Conservation Laws, second Edition, 1992, Springer

Richard H (1997). Mathematical Models, Prentice-Hall Inc.

Stig Larsson and Vidar Thomee (2005). Partial Differential Equations with Numerical Methods, Second Printing 2005, Springer-Verlag Berlin Heidelberg.

Received: 05 May 2010; Revised : 14 June 2010; Accepted: 03 March 2011. 